\title{
Spectral Stability Under Tunneling
}

\author{
Ph. Briet ${ }^{\star \star}$, J. M. Combes ${ }^{\star \star}$, and P. Duclos ${ }^{\star \star}$ \\ Centre de Physique Théorique ${ }^{\star}$, CNRS-Luminy, Case 907, F-13288, Marseille Cedex 09, France
}

\begin{abstract}
We study the spectral properties of multiple well Schrödinger operators on $\mathbb{R}^{n}$. We give in particular upper bounds on energy shifts due to tunnel effect and localization properties of wave packets. Our methods are based on Agmon type estimates for resolvents in classically forbidden regions and geometric perturbation theory. Our results are valid also for an infinite number of wells, arbitrary spectral type and in non-semi-classical regimes.
\end{abstract}

\section{Introduction}

The analysis of multiple well Schrödinger operators $H:=-g^{2} \Delta+V$ on $\mathscr{H}:=L^{2}\left(\mathbb{R}^{n}\right)$ is a new challenging aspect of the spectral theory for elliptic operators, linked to many problems of current interest like quasi-periodic potentials, composite media or disordered systems. It has attracted considerable attention in the last few years; for one dimensional problems let us mention in particular the work of Harrell [Ha1] using ODE methods, Jona-Lasinio, Martinelli and Scoppola [JMSc1] using stochastic mechanics and Combes, Duclos and Seiler [CDS1], where the use of perturbation methods were initiated. In dimensions larger than one there is in particular a remarkable amount of results from Helffer and Sjöstrand [HSj1-3] and Simon [Sim1-3]. These works concern the semiclassical regime for regular positive potentials with finitely many compact wells, having non-degenerate minima (see however [MaR] and references therein for results in the degenerate case). In such situations the leading asymptotics of energy shifts for the lowest eigenvalues under tunneling can be derived rigorously. One natural question is whether one can also obtain rigorous non-asymptotic estimates for arbitrary potentials; in other words is it possible to obtain general stability conditions both for the spectrum (upper bounds on energy shifts) and the localisation of wave functions. This is the problem which is investigated in this

\footnotetext{
* Laboratoire Propre, Centre National de la Recherche Scientifique

$\star \star$ Phymat, Université de Toulon et du Var
} 
paper; we show that it can be solved provided some separation parameter between wells is larger than some explicit constant depending only on the spectral properties of single well Hamiltonians. Our results are stated in a form which is general enough to accommodated all situations of interest, in particular those where the usual restrictions do not hold, e.g. finiteness of the number of wells, discreteness of the spectrum or semi-boundedness of the potential; it is clear that in many physical situations of interest as we shall see in Sect. VI, such aspects are not of purely academic interest.

Our main results are given in Sect. V. We give here a brief overview of their content; consider a compact energy interval $I:=\left[I^{-}, I^{+}\right]$such that one has a decomposition into "wells":

$$
V^{-1}\left(-\infty, I^{+}\right)=: \bigcup_{i>0} U_{i}
$$

(the $U_{i}$ are not necessarily connected). Let $d_{i j}$ denote Agmon's distance at energy $I^{+}$between $U_{i}$ and $U_{j}$ (see (II.4)) and $d_{i}:=\inf _{i \neq j} d_{i j}$. Let also $\tilde{d}_{i}:=\varepsilon_{i} d_{i}$ where $\varepsilon_{i} \in(0,1)$
satisfies

$$
\varepsilon_{i} d_{i}+\varepsilon_{j} d_{j} \leqq d_{i j} \text { for all } i \neq j .
$$

One introduces single well Hamiltonians $H_{i}:=-g^{2} \Delta+V_{i}$, where $V_{i}:=V$ on $U_{i}$ and $V_{i}:=\max \left(V, I^{+}\right)$outside $U_{i}$.

To state these results the following notations are used:

$$
\omega_{\delta}^{2}:=\sup _{E \in I}\left\{\max (V-E, 0), x \in \Omega_{\delta}^{(E)}\right\}, \quad \hat{\omega}_{\delta}:=\max \left(\omega_{\delta}, \sqrt{|I|}\right)
$$

where $\Omega_{\delta}^{(E)}$ denotes (see II.12) the set of points in the classically forbidden region at energy $E$ whose distance to the accessible one is smaller than $\delta$,

$$
\tilde{d}:=\inf _{\sigma\left(H_{i}\right) \cap I \neq \emptyset} \tilde{d}_{i}
$$

and $P(I)$ (respectively $P_{i}(I)$ ) the spectral projection for $H$ (respectively $H_{i}$ ) on the interval $I$.

Under some general assumptions on $V$ (see A.1, 2 given at the beginning of the next section) one has:

Theorem I.1. For any $\delta$ satisfying, $\forall i>0,2<\delta<\frac{\tilde{d}_{i}}{2}$, there exist constants $C_{1}, C_{2}$, and $C_{3}$ which depend only on $\delta$ such that:

i) If for all $i$

$$
\operatorname{dist}\left(I^{ \pm}, \sigma\left(H_{i}\right)\right) \geqq C_{1}(\delta) \omega_{\delta}^{2} e^{-2 \tilde{d}_{i}},
$$

then: $I^{ \pm} \in \varrho(H)$ (the resolvent set of $H$ ).

ii) If in addition for all $i$

$$
\Delta:=\inf _{i>0} \operatorname{dist}\left(I^{ \pm}, \sigma\left(H_{i}\right)\right)>C_{2}(\delta) \hat{\omega}_{\delta}^{2} \tilde{d}^{2} e^{-2 \tilde{d}},
$$

then $\operatorname{dim} P(I)=\sum_{i>0} \operatorname{dim} P_{i}(I)$.

iii) Under conditions $(\mathrm{I} .3,4)$ and the extra condition on $\delta, 2<\delta<\frac{d_{i}}{4}$, for each $\varphi_{i} \in P_{i}(I) \mathscr{H},\left\|\varphi_{i}\right\|=1$, there exists $\varphi \in P(I) \mathscr{H}$ such that

$$
\left\|\varphi-\varphi_{i}\right\| \leqq C_{3}(\delta)\left(\omega_{\delta} \Delta^{-1 / 2}+\omega_{\delta}^{2} \Delta^{-1}\right) \tilde{d} e^{-d_{i}} .
$$


The constants read as follows:

$$
C_{1}(\delta):=\frac{2(1+2 \delta)}{\delta^{2}(\delta-2)} e^{4 \delta}, \quad C_{2}(\delta):=432\left(\frac{\delta+3}{\delta-2}\right)^{4} e^{4 \delta}, \quad C_{3}(\delta):=16 e^{4 \delta} .
$$

Remarks I.2. i) The choice of the potential $V_{i}$ is to a large extent arbitrary; it is only necessary that for the partition of unity $\left(J_{i}\right)_{i \leq 0}$ separating the wells as introduced in Sect. IV one has $V_{i}=V$ on the support of $\bar{J}_{i}$ and $V_{i} \geqq V$ otherwise. Thus $\sigma\left(H_{i}\right) \cap I$ really depends on the local behaviour of $V$ near $U_{i}$ only.

ii) The conditions $2<\delta<\frac{\mathfrak{d}_{i}}{2}$ and $2<\delta<\frac{d_{i}}{4}$ are in fact conditions on the potential $V$. Although they are always fulfilled in most of the applications they look unnatural; they are required for technical reasons. In [BCD3] we drop this condition but an assumption on the finiteness of a kind of density of wells is then necessary.

This theorem will be proved in Sect. V. In Sect. II we derive the basic estimates for tunneling. There, a basic role is played by Agmon's metric which provides a natural perturbation parameter. These estimates concern resolvents localized in some domains of the classically forbidden region. As a byproduct we obtain in Sect. III decay properties of wave-packets with energy bounded above, extending those obtained by Agmon for proper eigenfunctions [Ag]. In Sect. IV we describe the geometric perturbative framework from which our results will be derived in a rather standard way in Sect. V. Finally, examples are briefly discussed in Sect. VI.

\section{Resolvent Estimates in the Classically Forbidden Region}

We present here a variant of Agmon's method [Ag] (see also [HSj1, 2]) to prove that resolvents of Schrödinger Hamiltonians admit classical bounds in the classically forbidden region up to corrections exponentially small in some distance measuring how far one is from the classically allowed regions. Although such estimates hold for more general differential operators of second order we shall only consider there Hamiltonians of the type

$$
H:=-g^{2} \Delta+V,
$$

where $V$ is a real potential and we assume:

Assumption A.1. $V=: V_{1}+V_{2}$ with $V_{1} \in L_{\mathrm{loc}}^{2}\left(\mathbb{R}^{n}\right), \quad V_{1} \geqq-C x^{2}-D$ and $V_{2}$ is $\Delta$-bounded with relative bound zero, $C$ and $D$ being two positive constants.

A1 implies that $H$ is essentially self-adjoint on $C_{0}^{\infty}\left(\mathbb{R}^{n}\right)[\mathrm{RS}]$.

The resolvents are defined by

$$
R(z):=(H-z)^{-1}, \quad z \in \varrho(H),
$$

where $E:=\operatorname{Re} z$ belongs to the energy interval $I:=\left[I^{-}, I^{+}\right]$.

In order to avoid irrelevant technicalities in particular with the definition of Agmon's metric (II.4) we also require

Assumption A.2. $(V-E)_{+}:=\max (V-E, 0) \in\left(L^{\infty} \cap C^{0}\right)\left(\mathbb{R}^{n}\right) \forall E \in I$. 
This assumption on $V$ can be in fact considerably weakened (see Remark II.5.1 below). Under (A.2) the classically forbidden region.

$$
G(E):=\{x, V(x)>E\}
$$

is open; we denote by $G_{c}(E)$ its complement. Agmon's metric at energy $E \in I$ is defined as

$$
d^{E} S^{2}:=g^{-1}(V-E)_{+} d x^{2}
$$

and $d^{E}(x, y)$ is the corresponding geodesic distance. We refer to $[\mathrm{Ag}]$ for details about properties of $d^{E}$ which will be used here.

Consider now an open set $\Omega \subset G(E)$ and define:

$$
\varrho_{\Omega}^{E}(x):=d^{E}(x, \Omega) .
$$

Then $\varrho_{\Omega}^{E}$ is a Lipschitz function and:

$$
g^{2}\left|\nabla \varrho_{\Omega}^{E}\right|^{2} \leqq(V-E)+\text { a.e. . }
$$

Obviously $\nabla \varrho_{\Omega}^{E}=0$ on $\Omega$ and $\varrho_{\Omega}^{E}$ has a constant value on $G_{c}(E)$ given by:

$$
d_{\Omega}^{E}:=d^{E}\left(G_{c}(E), \Omega\right) .
$$

Since $\Omega$ is open the positive quadratic form on $\mathscr{H}^{1}(\Omega)$ given by:

$$
a_{\Omega}^{E}[v]:=g^{2}\|\nabla v\|_{\Omega}^{2}+\left\|(V-E)_{+}^{1 / 2} v\right\|_{\Omega}^{2}
$$

is closed (see e.g. [Ad]) where we define

$$
\|u\|_{\Omega}^{2}:=\int_{\Omega}|u|^{2} d x
$$

and use the notation $\mathscr{H}^{1}(\Omega)$ for the set of the $u$ in $\mathscr{H}$ whose restriction to $\Omega$ is a.e. differentiable with $\nabla u \in L^{2}(\Omega)$.

Then there exists, by the second representation theorem [K], a positive selfadjoint operator $C_{\Omega}^{E}$ such that

$$
\begin{gathered}
\mathbf{D}\left(\Omega_{\Omega}^{E}\right):=\mathscr{H}^{1}(\Omega), \\
\left\|C_{\Omega}^{E} v\right\|^{2}:=a_{\Omega}^{E}[v] \quad \forall v \in \mathscr{H}^{1}(\Omega) .
\end{gathered}
$$

Lemma II.1. below shows that under our assumptions one has

$$
\mathbf{D}(H) \subset \mathscr{H}^{1}(\Omega) .
$$

We shall need the following elementary result

$$
\left\|C_{\Omega}^{E} e^{-e^{E}} u\right\|^{2}=\left\|C_{\Omega}^{E} u\right\|^{2} \quad \forall u \in \mathscr{H}^{1}(\Omega) .
$$

In the following we shall often drop, unless necessary, the indices $E$ and $\Omega$ in the previous notations for simplicity; when considering various sets $\Omega_{i}$ we shall also write $C_{i}$ for $C_{\Omega_{i}}^{E}$ etc.

The next lemma is a warm-up for the derivation of our main estimates:

Lemma II.1. Let $V$ satisfies (A.1), (A.2) and $z \in \varrho(H)$ satisfy

$$
\operatorname{Re} z \leqq E \text { for some } E \in I \text {. }
$$


Let $\Omega \subset G(E)$ be an open set such that $d_{\Omega}^{E}>0$; then $D(H) \subset \mathscr{H}^{1}(\Omega)$ and for all $\delta, 0<\delta$ $<d_{\Omega}^{E}$, one has:

$$
\left\|C_{\Omega}^{E} R(z)\right\|^{2} \leqq\|R(z)\|+e^{-2 d_{\Omega}^{E}} b(\delta)\left\|W_{\delta} R(z)\right\|^{2},
$$

where $W_{\delta}:=W_{\delta}^{(E)}$ is the restriction of $(V-E)_{+}^{1 / 2}$ to

$$
\Omega_{\delta}:=\Omega_{\delta}^{E}:=\left\{x \in G(E), d^{E}\left(x, G_{c}(E)\right)<\delta\right\}
$$

and $b(\delta):=\frac{1+2 \delta}{\delta^{2}} e^{2 \delta}$.

Proof. Given $\delta$ as above consider the function $\chi$ equal to 1 on $G(E) \backslash \Omega_{\delta}, 0$ outside $G(E)$ and $\chi(x):=\frac{d\left(x, G_{c}(E)\right)}{\delta}$ on $\Omega_{\delta}$; in particular $\chi$ is equal to 1 on $\Omega$ since $\delta<d_{\Omega}$. Then $\chi$ is a Lipschitz function and satisfies

$$
g^{2}|\nabla \chi|^{2} \leqq \frac{1}{\delta^{2}}(V-E)_{+} \text {a.e. on } \Omega_{\delta}
$$

whereas $\nabla \chi=0$ outside $\Omega_{\delta}$. Now let $\tilde{u} \in C_{0}^{\infty}$; one has the standard equality [Ag]:

$$
\|\nabla(\psi \tilde{u})\|^{2}=\operatorname{Re}\left\langle\nabla \tilde{u}, \nabla\left(\psi^{2} \tilde{u}\right)\right\rangle+\||\nabla \psi| \tilde{u}\|^{2}
$$

valid for any real and almost everywhere differentiable function $\psi$.

Choosing $\psi:=\chi e^{-\varrho}$ with $\varrho(x):=d(x, \Omega)$ gives:

$$
\begin{aligned}
\left\|C_{\Omega} \tilde{u}\right\|^{2} & \leqq g^{2}\left\|\nabla\left(\chi e^{-\varrho} \tilde{u}\right)\right\|^{2}+\left\|(V-E)_{+}^{1 / 2} \tilde{u}\right\|_{\Omega}^{2} \\
& \leqq \operatorname{Re}\left\langle\chi^{2} e^{-2 \varrho} \tilde{u},(H-z) \tilde{u}\right\rangle-\left\|(V-\operatorname{Re} z)_{+}^{1 / 2} \chi e^{-\varrho} \tilde{u}\right\|_{G(E) \backslash \Omega}^{2}+g^{2}\left\|\left|\nabla\left(\chi e^{-\varrho}\right)\right| \tilde{u}\right\|^{2} .
\end{aligned}
$$

From (II.13) one obtains easily the estimate:

$$
\left.g^{2}|| \nabla\left(\chi e^{-\varrho}\right)\right|^{2}-\left(\chi|\nabla \varrho| e^{-\varrho}\right)^{2} \mid \leqq \frac{1+2 \delta}{\delta^{2}} e^{-2 \varrho}\left(W_{\delta}^{(E)}\right)^{2}
$$

and (II.16) gives since $\operatorname{Re} z \leqq E$ :

$$
g^{2}\left\|\chi|\nabla \varrho| e^{-\varrho} \tilde{u}\right\|^{2} \leqq\left\|(V-\operatorname{Re} z)_{+}^{1 / 2} \chi e^{-\varrho} \tilde{u}\right\|^{2} .
$$

Inserting (II.16, 17) into (II.15) one gets:

$$
\left\|C_{\Omega} \tilde{u}\right\|^{2} \leqq \operatorname{Re}\left\langle\chi^{2} e^{-2 \varrho} \tilde{u},(H-z) \tilde{u}\right\rangle+\frac{1+2 \delta}{\delta^{2}}\left\|e^{-\varrho} W_{\delta}^{(E)} \tilde{u}\right\|^{2} .
$$

By continuity and by (A.1), (A.2) this inequality extends to any $\tilde{u}:=R(z) u, u \in \mathscr{H}$, giving

$$
\left\|C_{\Omega} R(z) u\right\|^{2} \leqq \operatorname{Re}\left\langle\chi^{2} e^{-2 \varrho} u, \tilde{u}\right\rangle+e^{-2 d_{\Omega}^{E}} b(\delta)\left\|W_{\delta}^{(E)} R(z) u\right\|^{2},
$$

where we used the fact that $\varrho \geqq d_{\Omega}^{E}-\delta$ on $\Omega_{\delta}$ by the triangle inequality; the proof of the lemma is easily completed with the Cauchy-Schwarz inequality.

Remark II.2. Notice (II.19) takes a very simple form if the support of $u$ lies in $G_{c}(E)$.

More generally the next lemma establishes decay properties of the Green's function between subsets of $G(E)$ and regions close to the wells: 
Lemma II.3. Under the assumptions of Lemma II.1 one has

$$
\left\|C_{\Omega}^{E} R(z) W_{\delta}^{(E)}\right\|^{2} \leqq e^{-2 d_{\Omega}^{E}}\left(e^{2 \delta} a+b(\delta) a^{2}\right),
$$

where

$$
a:=\left\|W_{\delta}^{(E)} R(z) W_{\delta}^{(E)}\right\| .
$$

Proof. We use (II.19) setting $u:=W_{\delta}^{(E)} v,\|v\|=1$; since $\chi \leqq 1$ on $\Omega_{\delta}$ one has:

$$
\operatorname{Re}\left\langle\chi^{2} e^{-2 \varrho} W_{\delta}^{(E)} v, R(z) W_{\delta}^{(E)} v\right\rangle \leqq e^{-2 d\left(\Omega, \Omega_{\delta}\right)} a .
$$

Since $d\left(\Omega, \Omega_{\delta}\right) \geqq d_{\Omega}^{E}-\delta$, (II.20) follows immediately.

Now we prove our main estimate:

Theorem II.4. Under the assumptions of Lemma II.1 the operator $C_{\Omega}^{E} R(z) C_{\Omega}^{E}$ has a bounded extension to $\mathscr{H}$ satisfying:

$$
\left\|C_{\Omega}^{E} R(z) C_{\Omega}^{E}\right\| \leqq 1+e^{-2 d_{\Omega}^{E}} b(\delta) a,
$$

where $a$ is given by (II.21) and $0<\delta<d_{\Omega}^{E}$.

Proof. We use again (II.19) setting now $u:=C_{\Omega} v$ with $v \in \mathscr{H}^{1}(\Omega),\|v\|=1$; then using (II.19) and the Cauchy-Schwarz inequality we obtain:

$$
\left\|C_{\Omega} R(z) C_{\Omega} v\right\|^{2} \leqq\left\|C_{\Omega} R(z) C_{\Omega} v\right\|+e^{-2 d_{\Omega}^{E}} b(\delta)\left\|W_{\delta}^{(E)} R(z) C_{\Omega} v\right\|^{2} .
$$

We estimate $\left\|W_{\delta}^{(E)} R(z) C_{\Omega} v\right\| \leqq\left\|C_{\Omega} R(\bar{z}) W_{\delta}^{(E)}\right\|$ using a rough form of (II.20):

$$
\left\|W_{\delta} R(z) C_{\Omega} v\right\|^{2} \leqq a+e^{-2 d_{\Omega}^{E}} b(\delta) a^{2} .
$$

Thus (II.24) reads $\alpha(\alpha-1) \leqq \beta(\beta+1)$ with $\alpha:=\left\|C_{\Omega} R(z) C_{\Omega} v\right\|$ and $\beta:=\mathrm{e}^{-2 d_{\Omega}^{E}} b(\delta) a$; this inequality remains valid for all $v \in \mathscr{H},\|v\|=1$, by the density of $\mathscr{H}^{1}(\Omega)$; this implies (II.23).

Remarks II.5. 1. It is clear from these proofs that the boundedness assumption (A.2) on $(V-E)_{+}$can be considerably weakened. In particular it is enough to assume that $W_{\delta}^{(E)}$ is bounded or even form bounded with respect to the Laplacian on $\Omega_{\delta}$. Notice also that in case $(V-E)_{-}$is bounded or $\Delta$-bounded the use of the characteristic function $\chi$ in the proof of Lemma II.1 and elsewhere can be avoided leading to a rather straightforward derivation of estimates of type (II.11) and (II.23). Finally notice that if $(V-E)_{+}$is only piecewise continuous (II.6) still holds.

2. It is easy to obtain from (II.23) some bounds on the resolvent kernel or its derivatives localized in some region of $G(E)$. Assume for example that $\alpha:=\inf _{\Omega}(V-E)>0$; then denoting by $\chi_{\Omega}$ the characteristic function of $\Omega$ one has

$$
\left\|\chi_{\Omega} R(z) \chi_{\Omega}\right\| \leqq \alpha^{-1 / 2}\left\|C_{\Omega} R(z) \chi_{\Omega}\right\| \leqq \alpha^{-1}\left\|C_{\Omega} R(z) C_{\Omega}\right\| \leqq \alpha^{-1}\left(1+e^{-2 d_{\Omega}^{E}} b(\delta) a\right),
$$

and also

$$
\left\|\chi_{\Omega} \nabla R(z) \chi_{\Omega}\right\| \leqq g^{-1}\left\|C_{\Omega} R(z) \chi_{\Omega}\right\| \leqq\left(\alpha g^{2}\right)^{-1 / 2}\left(1+e^{-2 d_{\Omega}^{E}} b(\delta) a\right) .
$$

Notice that in (II.25) the term $\alpha^{-1}$ is of purely classical nature whereas the exponentially small correction represents the quantum contribution.

3. All the estimates given above still hold if $R(z)$ is replaced by $R(z) P$, where $P$ is an orthonormal projection on some invariant subspace for $H$. 
4. In case there is no well for $H$ at energy $E \leqq I^{+}$, i.e. $V^{-1}(-\infty, E)=\emptyset$, then $\Omega_{\delta}$ in (II.26) is empty, hence $W_{\delta}^{(E)}$ can be taken equal to zero. Then all the results of this chapter become very simple; in particular one has

$$
\begin{gathered}
\left\|C_{\Omega}^{E} R(z)\right\|^{2} \leqq\|R(z)\|, \quad \operatorname{Re} z \leqq E, \\
\left\|C_{\Omega}^{E} R(z) C_{\Omega}^{E}\right\| \leqq 1 .
\end{gathered}
$$

\section{Decay Properties of Wave-Functions in the Classically Forbidden Region}

As an application of the estimates of Sect. II and in view of the perturbation theory to be developed later we consider now wave-functions in the range of $P(I)$, where $P$ denotes the spectral projection for $H, I:=\left[I^{-}, I^{+}\right]$is a given energy interval as in Sect. II, and show that they decay exponentially in $G\left(I^{+}\right)$in terms of Agmon's metric. This is well known for eigenfunctions of $H[\mathrm{Ag}]$ and is extended here to arbitrary wave-packets independently of the nature (discrete or continuous) of the spectrum of $H$. Our main result is stated with the notations of Lemma II.1 in:

Theorem III.1. Let $\varphi:=P\left(\left[\sigma^{-}, \sigma^{+}\right]\right) \varphi,\|\varphi\|=1$ with $\left[\sigma^{-}, \sigma^{+}\right] \subset I$ and $\left|I^{ \pm}-\sigma^{ \pm}\right| \geqq \Delta$ for some $\Delta>0$. Let $\Omega$ be an open subset of $G\left(I^{+}\right)$with $d_{\Omega}^{I^{+}}:=d^{I^{+}}\left(\Omega, G_{c}\left(I^{+}\right)\right)>0$. Then for all $\delta, 0<2 \delta<d_{\Omega}^{I^{+}}$, and $\beta>0$ one has:

$$
\left\|C_{\Omega}^{I^{+}} \varphi\right\| \leqq e^{-d_{\Omega}^{I^{+}}}\left[2 \pi^{-1} b(\delta)^{1 / 2} e^{\delta}\left\|W_{2 \delta}\right\|\left(|I| \beta^{-1}+2 s h^{-1}\left(\frac{2 \beta}{\Delta}\right)\right)\right],
$$

where $W_{2 \delta}:=W_{2 \delta}^{\left(I^{+}\right)}$.

As a preparation for the proof of this theorem let us define

$$
H_{0}:=-g^{2} \Delta+V_{0} \quad \text { with } \quad V_{0}:=\sup \left(I^{+}, V\right)
$$

so that $V_{0}$ has no well at energy $E<I^{+}$and the spectrum of $H_{0}$ is contained in $\left[I^{+}, \infty\right]$; we denote by $R_{0}$ the resolvent of $H_{0}$. Now let $\Omega \subset G\left(I^{+}\right)$be an open set such that $d_{\Omega}:=d_{\Omega}^{I^{+}}>0$; given $\delta, 0<\delta<d_{\Omega}$ consider the a.e. differentiable function $J_{0}$ equal to 1 in $G\left(I^{+}\right) \backslash \Omega_{\delta}$ (as defined by (II.12) with $E:=I^{+}$) and to $\frac{d\left(x, G_{c}\left(I^{+}\right)\right)}{\delta}$ outside. Then for $z \in \varrho\left(H_{0}\right) \cap \varrho(H)$ one has the geometric resolvent equation:

where

$$
J_{0} R(z)=R_{0}(z) J_{0}+R_{0}(z) M_{0} R(z),
$$

$$
\left\langle u, M_{0} v\right\rangle:=g^{2}\left(\left\langle\nabla u,\left(\nabla J_{0}\right) v\right\rangle-\left\langle\left(\nabla J_{0}\right) u, \nabla v\right\rangle\right)
$$

for $u, v \in C_{0}^{\infty}\left(\mathbb{R}^{n}\right)$. So (III.2) holds a priori only in the form sense on a suitable dense subset according to (A.1); it will follow from arguments presented below that it has an extension in the operator sense to $\mathscr{H}$.

Lemma III.2. Let $z \in \varrho\left(H_{0}\right) \cap \varrho(H)$ with $\operatorname{Re} z<I^{+}$; then $\forall \varphi \in \mathscr{H},\|\varphi\|=1$, one has for any $\delta, 0<2 \delta<d_{\Omega}^{I^{+}}$:

$$
\left\|C_{\Omega}^{I^{+}} R_{0}(z) M_{0} R(z) \varphi\right\| \leqq e^{-d_{\Omega}^{I^{+}}} e^{\delta} b(\delta)^{1 / 2}\left(\left\|W_{2 \delta} J_{0} R(z) \varphi\right\|+\left\|W_{2 \delta} R_{0}(z) J_{0} \varphi\right\|\right),
$$

where $W_{2 \delta}:=W_{2 \delta}^{\left(I^{+}\right)}$. 
Proof. Given $\delta$ as above consider the function $\chi$ equal to one on $G\left(I^{+}\right) \backslash \Omega_{2 \delta}$, to $\frac{d^{I^{+}}\left(x, G_{c}\left(I^{+}\right)\right)-\delta}{\delta}$ on $\Omega_{2 \delta} \backslash \Omega_{\delta}$ and zero elsewhere with $\Omega_{2 \delta}:=\Omega_{2 \delta}^{I^{+}}$and $\Omega_{\delta}:=\Omega_{\delta}^{I^{+}}$. Then using the same steps as those leading to (II.19) and the fact that $\chi=0$ on $\Omega_{\delta}$ one obtains

$$
\left\|C_{\Omega}^{I^{+}} R_{0}(z) u\right\|^{2} \leqq e^{-2 d_{s}^{I_{2}^{+}}} b(\delta) e^{2 \delta}\left\|W_{2 \delta} R_{0}(z) u\right\|^{2}
$$

for all $u$ with support in $\Omega_{\delta}$. Now it is easy to see that $C_{\Omega}^{I^{+}} R_{0}(z)$ and $W_{2 \delta} R_{0}(z)$ extend to bounded operators from $\mathscr{H}^{-1}\left(\mathbb{R}^{n}\right)$ to $\mathscr{H}$. This follows from the fact that their adjoints are bounded from $\mathscr{H}$ to $\mathscr{H}^{1}\left(\mathbb{R}^{n}\right)$ as a consequence e.g. of Lemma II.3 and positivity of $V_{0}$. Then (III.4) extends by continuity to $u \in \mathscr{H}^{-1}\left(\mathbb{R}^{n}\right)$ with support in $\Omega_{\delta}$; this covers the situation where $u:=M_{0} v$ with $v \in C_{0}^{\infty}\left(\mathbb{R}^{n}\right)$. Choosing in particular $v:=R(z) \varphi$, with $\varphi$ in the suitable dense subset of $\mathscr{H}$ one obtains:

$$
\left\|C_{\Omega}^{I^{+}} R_{0}(z) M_{0} R(z) \varphi\right\|^{2} \leqq e^{-2 d_{2}^{+}} b(\delta) e^{2 \delta}\left\|W_{2 \delta} R_{0}(z) M_{0} R(z) \varphi\right\|^{2},
$$

hence by (III.2):

$$
\left\|C_{\Omega}^{I^{+}} R_{0}(z) M_{0} R(z) \varphi\right\| \leqq e^{-d_{s}^{I^{+}}} e^{\delta} b(\delta)^{1 / 2}\left(\left\|W_{2 \delta} J_{0} R(z) \varphi\right\|+\left\|W_{2 \delta} R_{0}(z) J_{0} \varphi\right\|\right) .
$$

This extends again by continuity to any $\varphi \in \mathscr{H}$ since the right-hand side involves bounded operators.

We now give the proof of Theorem III.1:

Consider a contour as in Fig. 1 enclosing $\left[\sigma^{-}, \sigma^{+}\right]$with $\Delta \leqq \inf \left(I^{+}-\sigma^{+}\right.$, $\left.\sigma^{-}-I^{-}\right)$:

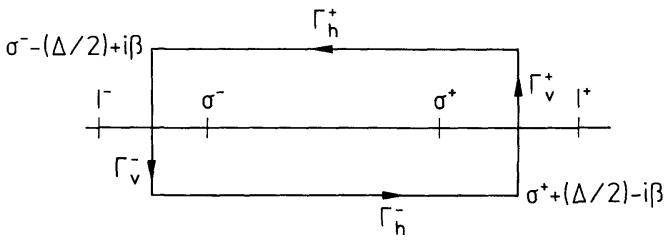

Fig. 1

then $J_{0} \varphi=-\frac{1}{2 i \pi} \int_{\Gamma} J_{0} R(z) \varphi d z=-\frac{1}{2 i \pi} \int_{\Gamma} R_{0}(z) M R(z) \varphi d z$ since $H_{0}$ has no spectrum inside $\Gamma$. So by Lemma III.2 one has

with

$$
\left\|C_{\Omega}^{I^{+}} \varphi\right\| \leqq C t e \int_{\Gamma}\left(\|R(z) \varphi\|+\left\|R_{0}(z) J_{0} \varphi\right\|\right)|d z|
$$

$$
C t e:=(2 \pi)^{-1} e^{-d_{\Omega 2}^{I}+} e^{\delta} b(\delta)^{1 / 2}\left\|W_{2 \delta}\right\| .
$$

The integral over $\Gamma_{h}^{+} \cup \Gamma_{h}^{-}$is easily estimated by $4|I| \beta^{-1}$. On $\Gamma_{v}^{+}$we set $z:=\sigma^{+}+\frac{\Delta}{2}$ $+i \eta, \eta \in[-\beta,+\beta]$, getting $\left\|R_{0}(z) J_{0} \varphi\right\| \leqq \frac{2}{\left(\Delta^{2}+4 \eta^{2}\right)^{1 / 2}}$, since $H_{0}$ has spectrum in
$\left[I^{+},+\infty\right)$, accordingly

$$
\int_{\Gamma_{v}^{+}}\left\|R_{0}(z) J_{0} \varphi\right\||d z| \leqq 2 \operatorname{sh}^{-1}\left(\frac{2 \beta}{\Delta}\right) .
$$


The same estimate holds for the integral over $\Gamma_{v}^{-}$. It also holds if $R_{0}(z) J_{0} \varphi$ is replaced by $R(z) \varphi$ since $\varphi=P\left(\left[\sigma^{-}, \sigma^{+}\right]\right) \varphi$; this concludes the proof of (III.1).

Remarks III.3. 1. As in Remark II.5, 2) one can obtain from (III.1) bounds on $\|\varphi\|_{\Omega}$ using

$$
\|\varphi\|_{\Omega} \leqq \alpha^{-1 / 2}\left\|C_{\Omega}^{I^{+}} \varphi\right\|,
$$

where we assume $\alpha:=\inf _{\Omega}\left(V-I^{+}\right)>0$.

2. A slightly different form of (III.5) will be useful in Sect. V; notice that in the proof of Theorem III.1 one can replace everywhere $I^{+}$by $E:=I^{+}-\frac{\Delta}{2}$, where $\Delta:=\inf \left(I^{+}-\sigma^{+}, \sigma^{-}-I^{-}\right)$. Since $\Omega \subset G\left(I^{+}\right)$one has $\inf _{\Omega}|V-E|>\frac{\Delta}{2}$; hence by (III.5):

$$
\|\varphi\|_{\Omega} \leqq\left(\frac{2}{\Delta}\right)^{1 / 2}\left\|C_{\Omega}^{E} \varphi\right\|
$$

Since $d_{\Omega}^{E} \geqq d_{\Omega}^{I^{+}}$one obtains from (III.1):

$$
\|\varphi\|_{\Omega} \leqq \frac{2 \sqrt{2}}{\pi} b(\delta)^{1 / 2} e^{\delta}\left\|W_{2 \delta}\right\|\left[e^{-d_{\Omega}^{I^{+}}} \Delta^{-1 / 2}\left(\beta^{-1}|I|+2 s h^{-1}\left(\frac{4 \beta}{\Delta}\right)\right)\right] .
$$

\section{The Geometric Perturbative Method}

We describe here the perturbative framework to relate the spectral properties of $H$ in a given energy range to those of single well (or cluster of wells) Hamiltonians. It consists essentially in introducing a suitable partition of unity separating wells and to use a localization formula for the resolvent of $H$ in terms of the resolvents for new Hamiltonians having the same symbol as $H$ in some regions only. As such it is closely related to the method followed by Helffer and Sjöstrand to establish decay properties of Green's functions [HSj2]; it is also connected to the well-known I.M.S. localization formula [Sig1]. In this perturbative method there is no natural small parameter; however it turns out that the perturbation introduced artificially by the decoupling can be chosen to be localized in the classically forbidden region and then its "size" can be analyzed with the estimates of Sect. II.

Then let $I:=\left[I^{-}, I^{+}\right]$be some energy domain we are interested in; assume

$$
V^{-1}\left(-\infty, I^{+}\right)=: \bigcup_{i>0} U_{i}
$$

where the wells $U_{i}$ are disjoint open sets; we do not assume that they are connected so that some of the $U_{i}$ can be a union of single (connected) wells. In order to separate the $U_{i}$ with a partition of unity we define:

$$
d_{i j}:=d^{I^{+}}\left(U_{i}, U_{j}\right), \quad d_{i}:=\inf _{j \neq i} d_{i j} .
$$

Then we introduce numbers $\varepsilon_{i}, 0<\varepsilon_{i}<1$ such that

$$
\varepsilon_{i} d_{i}+\varepsilon_{j} d_{j} \leqq d_{i j} \quad \forall i \neq j .
$$

The simplest choice is of course $\varepsilon_{i}:=\frac{1}{2}$ for all $i$, but there is some advantage in making different choices when some of the wells are "non-resonating" in the sense 
to be specified below. From (IV.3) it follows that the balls $B_{i}:=\left\{x, d^{I^{+}}\left(x, U_{i}\right)<\mathfrak{d}_{i}\right\}$ do not overlap where

$$
\tilde{d}_{i}:=\varepsilon_{i} d_{i} .
$$

We will assume in the following

Assumption A.3. For any $i>0, \tilde{d}_{i}>2 \delta$ for some $\delta>2$.

Then defining

where

$$
J_{i}(x):=j_{i}\left(d^{I^{+}}\left(x, U_{i}\right)\right),
$$

$$
j_{i}(d):=\left\{\begin{array}{lll}
0 & \text { if } & d \geqq \tilde{d}_{i} \\
1 & \text { if } & d \leqq \widetilde{d}_{i}-\delta \\
\frac{\tilde{d}_{i}-d}{\delta} & \text { if } & \tilde{d}_{i}-\delta \leqq d \leqq \tilde{d}_{i}
\end{array},\right.
$$

it follows that $J_{i}$ is a.e. differentiable and $\nabla J_{i}$ has support in $\bar{\Omega}_{i}$ with

$$
\Omega_{i}:=\left\{x, \tilde{d}_{i}-\delta<d^{I^{+}}\left(x, U_{i}\right)<\tilde{d}_{i}\right\} .
$$

According to (II.6) $\nabla J_{i}$ satisfies:

$$
g\left|\nabla J_{i}\right| \leqq \delta^{-1}\left(V-I^{+}\right)^{1 / 2} \text { a.e. }
$$

From (IV.3) it follows that $\sum_{i \geqq 1} J_{i} \leqq 1$; let

$$
J_{0}:=1-\sum_{i \geqq 1} J_{i}
$$

It is clear that the support of $J_{0}$ is in the classically forbidden region $G\left(I^{+}\right)$; in fact $\operatorname{supp} J_{0}=\left\{x, d\left(x, U_{i}\right) \geqq \tilde{d}_{i}-\delta \forall i>0\right\}$. Also from (IV.6), (IV.8) and the fact that the $J_{i}$ 's have a disjoint support it follows from (IV.8) that $\nabla J_{0}$ has support in the disjoint union:

and

$$
\Omega_{0}:=\bigcup_{i>0} \Omega_{i}
$$

$$
g\left|\nabla J_{0}\right| \leqq \delta^{-1}\left(V-I^{+}\right)^{1 / 2} .
$$

Now we introduce the well Hamiltonians

where

$$
H_{i}:=-\mathrm{g}^{2} \Delta+V_{i},
$$

and

$$
\forall i \geqq 1, \quad V_{i}(x):=\left\{\begin{array}{lll}
V(x), & \text { if } & x \in U_{i} \\
\max \left(V(x), I^{+}\right), & \text {if } & x \notin U_{i}
\end{array}\right.
$$

$$
V_{0}(x):=\max \left(V(x), I^{+}\right) .
$$

Notice that all the $V_{i}$ 's obey A.1 which implies that the $H_{i}$ 's are essentially selfadjoint on $C_{0}^{\infty}\left(\mathbb{R}^{n}\right)$.

The localization formula for resolvents expresses $R(z):=(H-z)^{-1}$ in terms of the $R_{i}(z):=\left(H_{i}-z\right)^{-1}$; it is based on the straightforward equality for $z \in \varrho(H)$ $\cap \varrho\left(H_{i}\right)$ :

$$
R(z) J_{i}=J_{i} R_{i}(z)+R(z) M_{i} R_{i}(z),
$$


where $M_{i}$ is defined in the form sense on $\mathscr{H}^{1}\left(\Omega_{i}\right)$ by:

$$
\left\langle u, M_{i} v\right\rangle:=g^{2}\left(\left\langle-\nabla u, \nabla J_{i} v\right\rangle+\left\langle\nabla J_{i} u, \nabla v\right\rangle\right) .
$$

So (IV.11) has to be understood in a form sense on suitable subsets of $\mathscr{H}$; however it follows from arguments similar to those used in the proof of Lemma III.2 that (IV.11) also holds in the operator sense on all of $\mathscr{H}$. It is convenient to sum all equalities (IV.11) in a simple compact form as follows: let

$$
\mathscr{H}_{\mathrm{d}}:=\underset{i \geqq 0}{\oplus} \mathscr{H}, \quad H_{d}:=\underset{i \geqq 0}{\oplus} H_{i},
$$

and $J$ be the bounded mapping from $\mathscr{H}_{d}$ to $\mathscr{H}$ defined by

Define also $\widetilde{J}$ as

$$
J\left(\underset{i \geqq 0}{\oplus} u_{i}\right):=\sum_{i \geqq 0} J_{i} u_{i} .
$$

$$
\widetilde{J}\left(\underset{i \geqq 0}{\oplus} u_{i}\right):=\sum_{i \geqq 0} \tilde{J}_{i} u_{i},
$$

where $\widetilde{J}_{i}$ is the characteristic function of support $J_{i}$; then

$$
J \widetilde{J}^{*}=\tilde{J} J^{*}=\mathbb{1} .
$$

Summing equalities (IV.11) and using (IV.14) one has:

where

$$
R(z) J=J R_{d}(z)+R(z) J\left(\tilde{J}^{*} M\right) R_{d}(z),
$$

$$
M\left(\underset{i \geqq 0}{\oplus} u_{i}\right):=\sum_{i \geqq 0} M_{i} u_{i} .
$$

This is the geometric resolvent equation; however it is not yet in a suitable form to be solved for $R(z)$ in terms of $R_{d}(z)$ (roughly speaking the kernel $\widetilde{J}^{*} M R_{d}(z)$ is not well-defined on $\mathscr{H}^{d}$ and even if it were, e.g. for smooth $J_{i}$, its norm is big compared to its spectral radius, thus leading to bad estimates). So we want to recast (IV.15) in a more convenient form; for this introduce the auxiliary space:

Then defining

$$
\mathscr{K}:=\left(\mathscr{H}_{d} \oplus \mathscr{H}_{d}\right)^{n} .
$$

$$
\mathscr{H}_{d}^{1}:=\underset{i \geqq 0}{\bigoplus} \mathscr{H}^{1}\left(\Omega_{i}\right)
$$

one considers the following mappings from $\mathscr{H}_{d}^{1}$ to $\mathscr{K}$ :

$$
\begin{gathered}
\left.\mathscr{M}_{1} \underset{i \geqq 0}{\oplus} u_{i}\right):=g \underset{i \geqq 0}{\oplus}\left(\alpha^{-1} \chi_{i} \nabla u_{i} \oplus \alpha\left(\nabla J_{i}\right) u_{i}\right), \\
\left.\mathscr{M}_{2} \underset{i \geqq 0}{\oplus} v_{i}\right):=g \underset{i \geqq 0}{\oplus}\left(\alpha\left(\nabla J_{i}\right) v_{i} \oplus \alpha^{-1} \chi_{i}\left(-\nabla v_{i}\right)\right),
\end{gathered}
$$

where $\chi_{i}$ is the characteristic function of $\Omega_{i}, \alpha:=\delta^{1 / 2}$ and $\delta$ is the positive number involved in the definition of the $J_{i}$ 's satisfying (A.3). Finally define $T: \mathscr{H}_{d} \rightarrow \mathscr{H}_{d}$ as:

$$
T\left(\underset{i \geqq 0}{\oplus} u_{i}\right):=\left(u_{0}-\sum_{j \geqq 1} \chi_{j} u_{j}\right) \oplus\left(\underset{i \geqq 1}{\oplus}\left(u_{i}-\chi_{i} u_{0}\right)\right) .
$$


Since the $\chi_{i}, i \geqq 1$, have disjoint supports by construction it is easy to show that $T$ is bounded and

$$
\|T\| \leqq 2 .
$$

Notice also that $T$ maps $\mathscr{H}_{d}^{1}$ into itself.

Lemma IV.1. One has:

$$
\tilde{J}^{*} M=\mathscr{M}_{2}^{*} \mathscr{M}_{1} T
$$

in the form sense on $\mathscr{H}_{d}^{1}$.

Proof. Let $\underset{i \geqq 0}{\oplus} u_{i}, \underset{i \geqq 0}{\oplus} v_{i} \in \mathscr{H}_{d}^{1}$; one has : $\left\langle\tilde{J} \underset{i}{\oplus} v_{i}, M \underset{j}{\oplus} u_{j}\right\rangle=\sum_{i j}\left\langle\tilde{J}_{i} v_{i}, M_{j} u_{j}\right\rangle$. This equality makes sense using the support properties of $\widetilde{J}_{i}$ and $\stackrel{i j}{\nabla} J_{i}$, namely for $i>0$ :

$$
\left\langle\tilde{J}_{i} v_{i}, M_{j} u_{j}\right\rangle=\left\{\begin{array}{cc}
0 & \text { if } j \neq i, 0 \\
\left\langle v_{i}, M_{i} u_{i}\right\rangle & \text { if } j=i \\
-\left\langle v_{i}, M_{i} \chi_{i} u_{0}\right\rangle & \text { if } j=0
\end{array} .\right.
$$

In this last equality we use $\mathscr{H}^{1}\left(\Omega_{0}\right) \subset \mathscr{H}^{1}\left(\Omega_{i}\right) \forall_{i} \neq 0$ and $\nabla J_{0}=-\nabla J_{i}$ on $\Omega_{i}$ according to the definition (IV.8) of $J_{0}$. One has also for the same reasons:

This gives:

$$
\left\langle\tilde{J}_{0} v_{0}, M_{j} u_{j}\right\rangle=-\left\langle v_{0}, M_{0} \chi_{j} u_{j}\right\rangle \text { if } j \neq 0 \text {. }
$$

$$
\left\langle\underset{i}{\oplus} v_{i}, \tilde{J}^{*} M\left(\underset{i}{\oplus} u_{i}\right)\right\rangle=\sum_{i}\left\langle v_{i}, M_{i} \omega_{i}\right\rangle
$$

with $\omega_{i}:=\left(T\left(\underset{j}{\oplus} u_{j}\right)\right)_{i}$. On the other hand from definitions (IV.17) one obtains immediately:

$$
\sum_{i}\left\langle v_{i}, M_{i} \omega_{i}\right\rangle=\left\langle\mathscr{M}_{2}\left(\underset{i}{\oplus} v_{i}\right), \mathscr{M}_{1}\left(\underset{i}{\oplus} \omega_{i}\right)\right\rangle
$$

which concludes the proof of (IV.20).

We define now the kernel

$$
K(z):=\mathscr{M}_{1} T R_{d}(z) \mathscr{M}_{2}^{*}
$$

acting on $\mathscr{K}$. We will show in the next lemma that $K(z)$ is bounded under a suitable assumption on $z$.

Lemma IV.2. Let $z \in \varrho\left(H_{d}\right), \operatorname{Re} z \leqq I^{+}$; then $K(z)$ is bounded on $\mathscr{K}$ and

$$
\|K(z)\| \leqq 2 \delta^{-1} \sup _{i \geqq 0}\left\|C_{\Omega_{i}}^{I^{+}} R_{i}(z) C_{\Omega_{\imath}}^{I^{+}}\right\| .
$$

Proof. One has by an elementary calculation:

$$
\left\|\mathscr{M}_{a}\left(\underset{i}{\oplus} u_{i}\right)\right\| \|^{2}=g^{2} \sum_{i}\left(\alpha^{-2}\left\|\nabla u_{i}\right\|_{\Omega_{i}}^{2}+\alpha^{2}\left\|\left|\nabla J_{i}\right| u_{i}\right\|^{2}\right) \quad \text { for } \underset{i}{\oplus} u_{i} \in \mathscr{H}_{d}^{1}, \quad a:=1,2
$$

Then according to (IV.7).

$$
\begin{aligned}
\left\|\mathscr{M}_{a}\left(\underset{i}{\oplus} u_{i}\right)\right\|^{2} & \leqq \sum_{i} \alpha^{-2} g^{2}\left\|\nabla u_{i}\right\|_{\Omega_{i}}^{2}+\alpha^{2} \delta^{-2}\left\|\left(V-I^{+}\right)^{1 / 2} u_{i}\right\|_{\Omega_{i}}^{2} \\
& \leqq \delta^{-1} \sum_{i}\left\|C_{i} u_{i}\right\|^{2}
\end{aligned}
$$


with our choice of $\alpha$ and $C_{i}=C_{\Omega_{i}}^{I^{+}}$. In particular one has from the definition (IV.18):

with

$$
\left\|\mathscr{M}_{a} T\left(\underset{i}{\oplus} v_{i}\right)\right\|^{2} \leqq \delta^{-1} \sum_{i}\left\|C_{i} \omega_{i}\right\|^{2}
$$

$$
\omega_{0}:=v_{0}-\sum_{j \geqq 1} \chi_{j} v_{j}, \quad \omega_{i}:=v_{i}-\chi_{i} v_{0}(i \geqq 1) .
$$

Since the $\chi_{i}$ have a disjoint support and $C_{i} \chi_{i}=C_{0} \chi_{i}$ one obtains easily

$$
\left\|\mathscr{M}_{a} T\left(\underset{i}{\oplus} v_{i}\right)\right\|^{2} \leqq 4 \delta^{-1} \sum_{i}\left\|C_{i} v_{i}\right\|^{2} \text { for } \underset{i}{\oplus} v_{i} \in \mathscr{H}_{d}^{1} .
$$

Now let $C_{d}$ be the diagonal operator on $H_{d}$ with domain $\mathscr{H}_{d}^{1}$ defined by

Then according to (IV.24)

$$
C_{d}\left(\underset{i>0}{\oplus} u_{i}\right)=\underset{i>0}{\oplus} C_{i} u_{i} .
$$

$$
\left\|\mathscr{M}_{1} T R_{d}(z) \mathscr{M}_{2}^{*} \psi\right\|^{2} \leqq 4 \delta^{-1}\left\|C_{d} R_{d}(z) \mathscr{M}_{2}^{*} \psi\right\|^{2}
$$

for all $\psi \in \mathscr{K}$ with components in $\mathscr{H}^{1}\left(\mathbb{R}^{n}\right)$.

On the other hand by (IV.23) and Theorem II.4 $\mathscr{M}_{2} R_{d}(\bar{z}) C_{d}$ has a bounded extension to $\mathscr{H}_{d}$ so that $C_{d} R_{d}(z) \mathscr{M}_{2}^{*}$ also does and by (IV.23),

Therefore

$$
\left\|C_{d} R_{d}(z) \mathscr{M}_{2}^{*}\right\|^{2}=\left\|\mathscr{M}_{2} R_{d}(\bar{z}) C_{d}\right\|^{2} \leqq \delta^{-1}\left\|C_{d} R_{d}(z) C_{d}\right\|^{2} .
$$

$$
\left\|\mathscr{M}_{1} T R_{d}(z) \mathscr{M}_{2}^{*} \psi\right\|^{2} \leqq 4 \delta^{-2}\left\|C_{d} R_{d}(z) C_{d}\right\|^{2}\|\psi\|^{2}
$$

for all $\psi \in \mathscr{K}$ which implies (IV.22).

We are now prepared to solve the geometric resolvent equation (IV.15):

Proposition IV.3. Let $z$ be in $\varrho\left(H_{d}\right)$ such that:
ii)

$$
\operatorname{Re} z \leqq I^{+}
$$$$
\|K(z)\|<1 \text {. }
$$

Then $z$ is in $\varrho(H)$ and

$$
R(z)=J R_{d}(z) \tilde{J}^{*}+J R_{d}(z) \mathscr{M}_{2}^{*}(1-K(z))^{-1} \mathscr{M}_{1} T R_{d}(z) \tilde{J}^{*} .
$$

Proof. Since $\varrho\left(H_{d}\right)$ is open and $K(z)$ is continuous on $\varrho\left(H_{d}\right)$ one chooses a ball $B(z, r):=\{\zeta \in \mathbb{C},|\zeta|<r\} \subset \varrho\left(H_{d}\right)$ such that $\|K(z)\|<1$ on $B(z, r)$; consider first $\operatorname{Im} \zeta \neq 0$ so that $\zeta \in \varrho(H) \cap \varrho\left(H_{d}\right)$; then multiplying (IV.15) on the right with $\mathscr{M}_{2}^{*}$ and using (IV.20) one obtains the equation

$$
R(\zeta) J \mathscr{M}_{2}^{*}=J R_{d}(\zeta) \mathscr{M}_{2}^{*}+R(\zeta) J \mathscr{M}_{2}^{*} K(\zeta)
$$

The operators $R(\zeta) J \mathscr{M}_{2}^{*}$ and $J R_{d}(\zeta) \mathscr{M}_{2}^{*}$ are well defined as it can be seen from the arguments used in the proof of Lemma IV.2. Solving for $R(\zeta) J \mathscr{M}_{2}^{*}$ and inserting the solution into (IV.15) with the help of (IV.20, 21) gives

$$
R(\zeta) J=J R_{d}(\zeta)+J R_{d}(\zeta) \mathscr{M}_{2}^{*}(1-K(\zeta))^{-1} \mathscr{M}_{1} T R_{d}(\zeta),
$$

from which (IV.27), with $z:=\zeta$, follows using (IV.14). Since $\|K(z)\|<1$ on $B(z, r)$, $(1-K(z))^{-1}$ is bounded analytic on $B(z, r)$ and so does the right-hand side of (IV.27); therefore $R(\zeta)$ is bounded analytic on $B(z, r)$; in particular $z$ belongs to $\varrho(H)$ and (IV.28), with $\zeta:=z$, as well as (IV.27) hold. 


\section{Spectral Stability Properties Under Tunneling}

We are now ready to prove our main results; let us show first the

Lemma V.1. Let $z$ be in $\varrho\left(H_{d}\right)$ such that:

i) $\operatorname{Re} z \leqq I^{+}$,

ii) for some $\delta$ satisfying (A.3) and some $\gamma \geqq 1$ :

where

$$
\forall i>0, \quad \gamma C_{1}(\delta) \omega_{\delta}^{2}\left\|R_{i}(z)\right\|<e^{2 \tilde{d}_{i}},
$$

Then one has

$$
C_{1}(\delta)=\frac{2(1+2 \delta)}{\delta^{2}(\delta-2)} e^{4 \delta} \quad \text { with } \quad \omega_{\delta}=\sup _{E \in I}\left\|W_{\delta}^{(E)}\right\| .
$$

$$
\begin{array}{r}
\|K(z)\|<\frac{2 \gamma+\delta-2}{\gamma \delta} \leqq 1, \\
z \in \varrho(H) \text { and (IV.27) holds. }
\end{array}
$$

Proof. By Proposition IV.3 it is enough to show that $\|K(z)\|<\frac{2 \gamma+\delta-2}{\gamma \delta}$ since $\frac{2 \gamma+\delta-2}{\gamma \delta} \leqq 1$ due to $\delta>2$ and $\gamma \geqq 1$; applying Lemma IV.2 it is sufficient to show that for all $i$ :

$$
\left\|C_{\Omega_{\mathrm{r}}}^{I^{+}} R_{i}(z) C_{\Omega_{\mathrm{\imath}}}^{I^{+}}\right\|<\frac{2 \gamma+\delta-2}{2 \gamma} .
$$

In case $i=0$ this follows immediately from the conditions $\delta>2, \gamma \geqq 1$ and (II.27). For $i \neq 0$ we apply Theorem II.4; then (V.4) holds provided

$$
1+e^{-2 d^{I^{+}}\left(U_{v}, \Omega_{\imath}\right)} b(\delta) a_{i}<\frac{2 \gamma+\delta-2}{2 \gamma} .
$$

Here $a_{i}=\left\|W_{i, \delta} R_{i}(z) W_{i, \delta}\right\|$ and $W_{i, \delta}$ is the restriction of $\left(V_{i}-I^{+}\right)^{1 / 2}$ to $\Omega_{\delta} \cap \operatorname{Supp} J_{i}$ (see Lemma II. 2 and notice that under A.3 its assumptions are satisfied). We use the rough estimate $a_{i} \leqq\left\|R_{i}(z)\right\| \omega_{\delta}^{2}$ and observe that with the definition (IV.6) of $\Omega_{i}$ one has $d^{I^{+}}\left(U_{i}, \Omega_{i}\right)=\overline{\bar{d}}_{i}-\delta$. So (V.5) holds if

$$
1+e^{-2 \tilde{d}_{i}} e^{2 \delta} b(\delta) \omega_{\delta}^{2}\left\|R_{i}(z)\right\|<\frac{2 \gamma+\delta-2}{2 \gamma},
$$

which is just the condition (V.1).

Lemma V.2. Let $E \in I$ be such that one has with $C_{1}(\delta)$ defined by (V.2):

$\exists \delta$ satisfying (A.3), $\forall i>0, \quad \operatorname{dist}\left(E, \sigma\left(H_{i}\right)\right)>C_{1}(\delta) \omega_{\delta}^{2} e^{-2 \tilde{d}_{i}}$, then $E \in \varrho(H)$.

Proof. This is an obvious byproduct of Lemma V.1.

An immediate consequence is

Theorem V.3 (Stability of gaps). Assume(V.7) holds for $E=I^{ \pm}$and that $\sigma\left(H_{i}\right) \cap I=\emptyset$ for all $i>0$; then $I \subset \varrho(H)$. 
Proof. Each point of $I$ satisfies (V.7) and therefore belongs to $\varrho(H)$.

Our next theorem gives upper bounds on energy shifts due to the interaction between wells; we will need the following:

Definition V.4. A well $U_{i}$ is said to be resonant in $I$ if $\sigma\left(H_{i}\right) \cap I$ is non-empty.

We denote by $R$ the set of resonant wells in $I$, by $N R$ the non resonant ones. One has:

Theorem V.5. (Upper bound on energy shifts). Assume (V.7) holds for $E:=I^{ \pm}$with some $\delta$; let $\sigma_{i}:=\sigma\left(H_{i}\right) \cap I, i \in R$, and

$$
\begin{aligned}
& E^{+}:=\sup _{i \in R}\left(\sigma_{i}^{+}+C_{1}(\delta) \omega_{\delta}^{2} e^{-2 \tilde{d}_{i}}\right), \\
& E^{-}:=\inf _{i \in R}\left(\sigma_{i}^{-1}-C_{1}(\delta) \omega_{\delta}^{2} e^{-2 \tilde{d}_{i}}\right) .
\end{aligned}
$$

Then $\left(E^{+}, I^{+}\right]$and $\left[I^{-}, E^{-}\right)$are subsets of $\varrho(H)$.

Proof. Any $E$ in these intervals obviously satisfies $\operatorname{dist}\left(E, \sigma\left(H_{i}\right)\right)>C_{1}(\delta) \omega_{\delta}^{2} e^{-2 \tilde{d}_{t}}$ when $i \in N R$. If $i \in R$ on one hand, $\operatorname{dist}\left(E, \sigma\left(H_{i}\right) \backslash \sigma_{i}\right)>C_{1}(\delta) \omega_{\delta}^{2} e^{-2 \tilde{d}_{1}}$, since this is true by assumption on $I^{ \pm}$and on the other hand, from the assumption on $E^{ \pm}, \operatorname{dist}\left(E, \sigma_{i}\right)$ $>C_{1}(\delta) \omega_{\delta}^{2} e^{-2 \tilde{d}_{t}}$. Thus for such $E(\mathrm{~V} .7)$ holds which by Lemma V.2 shows that they belong to $\varrho(H)$.

Remarks V.6. 1. Obviously Theorem V.5 becomes meaningful only if one shows that $\operatorname{dim} P(I)=\operatorname{dim} P\left(E^{-}, E^{+}\right)=\sum_{i \in R} \operatorname{dim} P_{i}(I)$; this will be a consequence of
Theorem I.1.

2. One can see from the above results that there is some advantage in leaving open the possibility to choose $\varepsilon_{i}$ different from $1 / 2$ in the definition (IV.4) of $\tilde{d}_{i}$. In particular if $i \in N R$ a condition like (V.7) can be satisfied in general for small choices of $\varepsilon_{i}$. Then if $j \in R$ and the nearest neighbour of $U_{j}$ is a non-resonant well one can choose $\varepsilon_{j}$ larger than $1 / 2$ thus providing better estimates on energy shifts through Theorem V.5. The simplest case where this can occur is the nonsymmetric double well if only one well, say $U_{1}$, is resonant in $I$. Assume that $I:=\left[E^{-}, E^{+}\right]$as given by (V.8); if $\varepsilon_{1}$ can be chosen close to 1 then Theorem V.5 says that the energy shift due to the interaction with $U_{2}$ is of the order of $e^{-2 d_{1}}$, whereas it is well-known that it is proportional only to $e^{-d_{1}}$ in the symmetric double well. There is another situation where this flexibility in the choice of $\varepsilon_{i}$ can be useful, namely when some $d_{i}$ are large for some $i$. There again (V.7) can be satisfied for small $\varepsilon_{i}$ thus allowing larger other choices of $\varepsilon_{j}, j \neq i$, satisfying (IV.3).

Our next theorem exhibits the localisation properties of states in the range of $P\left[E^{-}, E^{+}\right]$with $E^{ \pm}$as defined in Theorem V.5:

Theorem V.7 (Stability of localization). Assume that

$$
\exists \delta \text { satisfying (A.3), } \quad \forall i>0, \quad \operatorname{dist}\left(I^{ \pm}, \sigma\left(H_{i}\right)\right)>2 C_{1}(\delta) \omega_{\delta}^{2} e^{-2 \tilde{d}_{i}},
$$


where $C_{1}(\delta)$ is defined by (V.2) and let $\Delta:=\inf _{i>0} \operatorname{dist}\left(I^{ \pm}, \sigma\left(H_{i}\right)\right)$; then with $P:=P\left[E^{-}, E^{+}\right]$and $P_{d}:=\bigoplus_{i \in R} P_{i}(I)$ one has:

i) For any $\varphi_{d} \in P_{d} \mathscr{H}_{d},\left\|\varphi_{d}\right\|=1$, there exists $\varphi \in P \mathscr{H}$ such that

$$
\forall \beta>0, \quad\left\|\varphi-J \varphi_{d}\right\| \leqq C(\delta) \frac{\omega_{\delta} e^{-\tilde{d}}}{\Delta^{1 / 2}}\left(|I| \beta^{-1}+2 s h^{-1}\left(\frac{2 \beta}{\Delta}\right)\right),
$$

where $\tilde{d}:=\inf _{i \in R} \tilde{d}_{i}$ and

$$
C(\delta):=\frac{16 \sqrt{2}}{\pi} \frac{3 \delta-2}{\delta-2} \frac{\sqrt{(\delta+2)(\delta+1)}}{\delta(\delta-2)} e^{2 \delta} .
$$

ii) Conversely for any $\varphi \in P \mathscr{H},\|\varphi\|=1$, there exists $\varphi_{d} \in P_{d} \mathscr{H}_{d}$ such that

$$
\forall \beta>0, \quad\left\|\varphi_{d}-J^{*} \varphi\right\| \leqq C(\delta) \frac{\omega_{\delta} e^{-\tilde{d}}}{\Delta^{1 / 2}}\left(|I| \beta^{-1}+2 \operatorname{sh}^{-1}\left(\frac{2 \beta}{\Delta}\right)\right) .
$$

Proof. Consider the contour $\Gamma$ of Fig. 2 where $\sigma_{i}:=\sigma\left(H_{i}\right) \cap I$; then $\Gamma \subset \varrho\left(H_{d}\right) \cap \varrho(H)$ by condition (V.9) and Lemma V.2, and therefore the resolvent equality (IV.28) holds for all $z$ of $\Gamma$. According to it:

$$
P J=J P_{d}+S,
$$

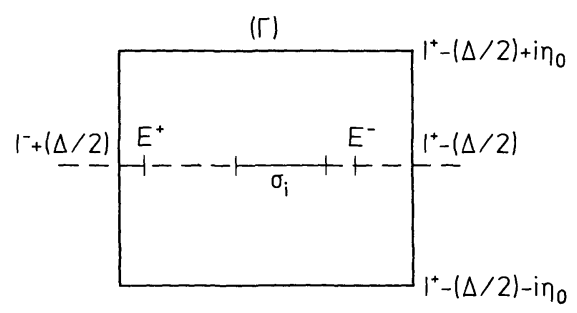

Fig. 2

where

$$
S:=-\frac{1}{2 i \pi} \int_{\Gamma} J R_{d}(z) \mathscr{M}_{2}^{*}(1-K(z))^{-1} \mathscr{M}_{1} T R_{d}(z) d z
$$

Let $K(z):=K_{r}(z)+K_{s}(z)$ be the decomposition of $K(z)$ into a regular and singular part inside $\Gamma$ :

$$
K_{r}(z):=\mathscr{M}_{1} T R_{d}(z)\left(1-P_{d}\right) \mathscr{M}_{2}^{*}, \quad K_{s}(z):=\mathscr{M}_{1} T R_{d}(z) P_{d} \mathscr{M}_{2}^{*} .
$$

Both $K_{r}(z)$ and $K_{s}(z)$ satisfy the same norm estimate (V.3) as $K(z)$ since the resolvents $R_{i}(z)$ are suitably restricted to the corresponding invariant subspaces. In particular according to the Remark II.5,3) and to Lemma V.1, with $\gamma:=2$, one sees easily that not only

$$
\forall z \in \Gamma, \quad\|K(z)\|<\frac{\delta+2}{2 \delta},
$$

but the same estimate also holds for $\left\|K_{r}(z)\right\|$ inside $\Gamma$; hence $\left(1-K_{r}(z)\right)^{-1}$ is holomorphic there. Now to the decomposition

$$
(1-K(z))^{-1}=\left(1-K_{r}(z)\right)^{-1}+(1-K(z))^{-1} K_{s}(z)\left(1-K_{r}(z)\right)^{-1}
$$


valid for $z$ of $\Gamma$ there corresponds

where e.g.

$$
S=S_{r}+S_{s}
$$

$$
S_{r}:=-\frac{1}{2 i \pi} \int_{\Gamma} J R_{d}(z) \mathscr{M}_{2}^{*}\left(1-K_{r}(z)\right)^{-1} \mathscr{M}_{1} T R_{d}(z) d z
$$

Decomposing $R_{d}(z)=R_{d}(z) P_{d}+R_{d}(z)\left(1-P_{d}\right)$ it follows from holomorphy of $\left(1-K_{r}(z)\right)^{-1}$ inside $\Gamma$ that in the corresponding decomposition of $S_{r}$ at least one singular resolvent $R_{d}(z) P_{d}$ has to appear. This provides an estimate

$$
\begin{aligned}
\left\|S_{r}\right\| \leqq & \frac{\delta}{\pi(\delta-2)} \int_{\Gamma}\left(\left\|\mathscr{M}_{2} R_{d}(z) J^{*}\right\|\left\|\mathscr{M}_{1} T P_{d} R_{d}(z)\right\|\right. \\
& \left.+\left\|\mathscr{M}_{2} P_{d} R_{d}(z) J^{*}\right\|\left\|\mathscr{M}_{1} T R_{d}(z)\right\|\right)|d z|
\end{aligned}
$$

According to (IV.23) and (IV.24) one obtains

$$
\left\|S_{r}\right\| \leqq \frac{4}{\pi(\delta-2)} \int_{\Gamma}\left\|C_{d} R_{d}(z)\right\|\left\|C_{d} P_{d} R_{d}(z)\right\||d z|,
$$

where $C_{d}$ is defined by (IV.25) and we used $\left\|J^{*}\right\| \leqq 1$. Applying (II.26) and Lemma II.1 one has

$$
\forall z \in \Gamma, \quad\left\|C_{d} R_{d}(z)\right\|^{2} \leqq \sup \left\{\left\|R_{0}\right\|, \sup _{i>0}\left\|R_{i}(z)\right\|\left(1+e^{-2 \tilde{d}_{i}} e^{2 \delta} b(\delta) \omega_{\delta}^{2}\left\|R_{i}(z)\right\|\right\},\right.
$$

which gives for $\eta_{0}$ large enough and since $\frac{\delta+2}{4}>1$,

$$
\left\|C_{d} R_{d}(z)\right\|^{2}<\frac{\delta+2}{4} \sup _{i \geqq 0}\left\|R_{i}(z)\right\| .
$$

The integral over the horizontal sides of $\Gamma$ can be made arbitrarily small by choosing $\eta_{0}$ large. On the vertical sides one has the spectral estimates

$$
\left\|R_{i}\left(I^{ \pm} \mp \frac{\Delta}{2}+i \eta\right)\right\| \leqq 2\left(\Delta^{2}+4 \eta^{2}\right)^{-1 / 2} \text { for } i \geqq 0
$$

hence:

and

$$
\left\|C_{d} P_{d} R_{d}(z)\right\| \leqq 2\left\|C_{d} P_{d}\right\|\left(\Delta^{2}+4 \eta^{2}\right)^{-1 / 2}
$$

$$
\left\|S_{r}\right\| \leqq \frac{8 \sqrt{2}}{\pi} \frac{\sqrt{\delta+2}}{\delta-2}\left\|C_{d} P_{d}\right\| \int_{-\infty}^{+\infty}\left(\Delta^{2}+4 \eta^{2}\right)^{-3 / 4} d \eta
$$
On the other hand applying Theorem III. 1 with $\frac{\delta}{2}$ instead of $\delta\left(\right.$ since $d_{\Omega_{i}}^{I^{+}}=\tilde{d}_{i}-\delta>\delta$
by (A.3)) gives

$$
\forall \beta>0, \quad\left\|C_{d} P_{d}\right\| \leqq 4(\pi \delta)^{-1}(1+\delta)^{1 / 2} e^{2 \delta} \omega_{\delta} e^{-\tilde{d}}\left(|I| \beta^{-1}+2 s h^{-1}\left(\frac{2 \beta}{\Delta}\right)\right) .
$$

Hence inserting (V.18) into (V.17), one has:

$\forall \beta>0, \quad\left\|S_{r}\right\| \leqq \frac{32 \sqrt{2}}{\pi} \frac{\sqrt{(\delta+2)(\delta+1)}}{\delta(\delta-2)} e^{2 \delta} \frac{\omega_{\delta} e^{-\tilde{d}}}{\Delta^{1 / 2}}\left(|I| \beta^{-1}+2 s h^{-1}\left(\frac{2 \beta}{\Delta}\right)\right)$. 
We now consider $S_{s}$; then by (V.14) and (V.15):

$$
\left\|S_{s}\right\| \leqq \frac{2}{\pi}\left(\frac{\delta}{\delta-2}\right)^{2} \int_{\Gamma}\left\|J R_{d}(z) \mathscr{M}_{2}^{*}\right\|\left\|K_{s}(z)\right\|\left\|\mathscr{M}_{1} T R_{d}(z)\right\||d z| .
$$

We estimate $\left\|K_{s}(z)\right\|$ with (IV.21) and (IV.22); again

$$
\left\|K_{s}(z)\right\| \leqq\left\|\mathscr{M}_{2} P_{d}\right\|\left\|\mathscr{M}_{1} T R_{d}(z)\right\| \leqq 2 \delta^{-1}\left\|C_{d} P_{d}\right\|\left\|C_{d} R_{d}(z)\right\| ;
$$

then using the same arguments as before one has

$$
\begin{aligned}
\left\|S_{s}\right\| & \leqq \frac{8}{\pi(\delta-2)^{2}}\left\|C_{d} P_{d}\right\| \int_{\Gamma}\left\|C_{d} R_{d}(z)\right\|^{3}|d z| \\
& \leqq \frac{4 \sqrt{2}(\delta+2)^{3 / 2}}{\pi(\delta-2)^{2}}\left\|C_{d} P_{d}\right\| \int_{-\infty}^{+\infty}\left(\Delta^{2}+4 \eta^{2}\right)^{-3 / 4} d \eta
\end{aligned}
$$

which gives with (V.18):

$$
\forall \beta>0, \quad\left\|S_{s}\right\| \leqq \frac{16 \sqrt{2}}{\pi} \frac{\sqrt{(\delta+2)(\delta+1)}}{\delta(\delta-2)} \frac{\delta+2}{\delta-2} \frac{\omega_{\delta} e^{-\tilde{d}}}{\Delta^{1 / 2}}\left(|I| \beta^{-1}+2 s h^{-1}\left(\frac{2 \beta}{\Delta}\right)\right) .
$$

Finally adding the two estimates on $S_{r}$ and $S_{s}$ gives:

$$
\forall \beta>0, \quad\left\|P J-J P_{d}\right\| \leqq C(\delta) \frac{\omega_{\delta} e^{-\tilde{d}}}{\Delta^{1 / 2}}\left(|I| \beta^{-1}+2 s h^{-1}\left(\frac{2 \beta}{\Delta}\right)\right)
$$

with $C(\delta)$ given by (V.11) which obviously proves i) and ii) of the theorem.

Proof of Theorem I.1. i) Follows from Lemma V.2. On the other hand it follows from Theorem V.5 that $P(I)=P\left[E^{-}, E^{+}\right]=: P$; we shall show that under (I.4) the following mappings:

$$
\begin{gathered}
P_{d} \mathscr{H}_{d} \ni \varphi_{d} \rightarrow P J \varphi_{d}=\varphi \in P \mathscr{H}, \\
P \mathscr{H} \ni \varphi \rightarrow P_{d} J^{*} \varphi=\varphi_{d} \in P_{d} \mathscr{H}_{d},
\end{gathered}
$$

are injective which will show ii). Consider the first one; from the straightforward inequality

$$
\forall 0<\varepsilon<1, \quad P_{d} J^{*} P J P_{d} \geqq(1-\varepsilon)\left[P_{d}-\oplus_{i \in R} P_{i}\left(1-J_{i}^{2}\right) P_{i}-\varepsilon^{-1} S^{*} S\right]
$$

it is sufficient to show that

$$
\|S\|^{2}+\left\|\underset{i \in R}{\oplus} \sqrt{1-J_{i}^{2}} P_{i}\right\|^{2}<1
$$

Applying (III.6), with $\frac{\delta}{2}$ instead of $\delta$, one has:

$$
\forall \beta>0, \quad\left\|\oplus_{i \in R} \sqrt{1-J_{i}^{2}} P_{i}\right\| \leqq \widetilde{C}(\delta) \frac{\omega_{\delta} e^{-\tilde{d}}}{\Delta^{1 / 2}}\left[|I| \beta^{-1}+2 s h^{-1}\left(\frac{4 \beta}{\Delta}\right)\right]
$$

for a constant $\tilde{C}(\delta):=\frac{4 \sqrt{2}}{\pi} \frac{\sqrt{1+\delta}}{\delta} e^{2 \delta}$. 
Similarly for the second mapping one has

$$
\forall 0<\varepsilon<1, \quad P J P_{d} J^{*} P \geqq(1-\varepsilon)\left[P-\sum_{i \in R} P\left(1-J_{i}^{2}\right) P-S S^{*}\right],
$$

which shows that it will be injective under the sufficient condition:

$$
\left\|\left(\sum_{i \in R} 1-J_{i}^{2}\right)^{1 / 2} P\right\|^{2}+\|S\|^{2}<1
$$

According to the support of the $J_{i}$ 's one has the analogue of (V.22):

$$
\forall \beta>0, \quad\left\|\left(\sum_{i \in R} 1-J_{i}^{2}\right)^{1 / 2} P\right\|^{2} \leqq \tilde{C}(\delta) \frac{\omega_{\delta} e^{-\tilde{d}}}{\Delta^{1 / 2}}\left[|I| \beta^{-1}+2 s h^{-1}\left(\frac{4 \beta}{\Delta}\right)\right] .
$$

It will be convenient to use, for $\alpha:=1,2$,

$$
2 \operatorname{sh}^{-1}\left(\frac{2 \alpha \beta}{\Delta}\right) \leqq 2 \ln \left(1+\frac{4 \alpha \beta}{\Delta}\right) \leqq 4 \tilde{d}+\frac{8 \alpha \beta}{\Delta} e^{-2 \tilde{d}},
$$

which gives after optimising over $\beta$,

$$
\exists \beta>0, \quad|I| \beta^{-1}+2 \operatorname{sh}^{-1}\left(\frac{2 \alpha \beta}{\Delta}\right) \leqq 4 \tilde{d}+4 \sqrt{2 \alpha} \frac{\sqrt{|I|} e^{-\tilde{d}}}{\Delta^{1 / 2}} .
$$

Setting $X:=\max \left(\sqrt{|I|}, \omega_{\delta}\right) \frac{d}{2 \delta} \frac{e^{-\tilde{d}}}{\Delta^{1 / 2}}$, one sees using $(\mathrm{V} .20,22,25)$ that tofulfill(V.21) and (V.23) it is sufficient to have

$$
4 \hat{C}(\delta) X \frac{2 \delta}{\tilde{d}}\left[\tilde{d}+\frac{2 \sqrt{2} \delta}{\tilde{d}} X\right]<1
$$

with $\widehat{C}(\delta)^{2}:=C(\delta)^{2}+2 \widetilde{C}(\delta)^{2}$.

By (A.3), (V.26) is a consequence of

$$
4 \sqrt{2} \hat{C}(\delta) X[\sqrt{2} \delta+X]<1
$$

Solving (V.27) with respect to $X$ shows that (V.26) is true if

$$
X=\max \left(\sqrt{|I|}, \omega_{\delta}\right) \frac{d}{2 \delta} \frac{e^{-\tilde{d}}}{\Delta^{1 / 2}} \leqq\left(\frac{9}{2} \hat{C}(\delta) \delta\right)^{-1}
$$

which is nothing but the second part of condition (I.4) of Theorem I.1, if one sets $C_{2}(\delta):=\left(\frac{9}{2} \hat{C}(\delta)\right)^{2}$. In fact we have chosen a bigger $C_{2}$ which has a simpler form.

We now come to the proof of part iii) of Theorem I.1 which is a refinement of Theorem V.7i). Consider a new function $J_{i}$ defined as the one introduced in (IV.5) with the choice $\tilde{d}_{i}:=d_{i}-\delta$ for some $\delta, 2<\delta<\frac{d_{i}}{4}$ (of course we do not expect that (IV.3) will hold any longer in general but this does not matter here). Then the resolvent equation (IV.11) holds; it is convenient to rewrite it using the factorisation method of Sect. IV (see (IV.20)) as

$$
R(z) J_{i}=J_{i} R_{i}(z)+R(z) \mathscr{M}_{2}^{(i)^{*}} \mathscr{M}_{1}^{(i)} R_{i}(z),
$$


where $\mathscr{M}_{a}^{(i)}: \mathscr{H} \rightarrow \mathscr{H} \oplus \mathscr{H}, a:=1,2$ is the $i^{\text {th }}$ component of the operator $\mathscr{M}_{a}$ as given by (IV.17). As in (IV.23) one has

$$
\left\|\mathscr{M}_{a}^{(i)} u\right\| \leqq \delta^{-1 / 2}\left\|C_{i} u\right\|
$$

Given $\varphi_{i} \in P_{i}(I) \mathscr{H},\left\|\varphi_{i}\right\|=1$, let $\varphi:=P J_{i} \varphi_{i}$; then

$$
\varphi=J_{i} \varphi_{i}+\psi
$$

where, with $\hat{R}(z):=R(z)(1-P)$, one has

$$
\|\psi\| \leqq \frac{1}{2 \pi} \int_{\Gamma}\left\|\hat{R}(z) \mathscr{M}_{2}^{(i)^{*}} \mathscr{M}_{1}^{(i)^{*}} R_{i}(z) \varphi_{i}\right\||d z|
$$

and $\Gamma$ is as in Fig. 1 with $\sigma^{ \pm}:=\sigma_{i}^{ \pm}$. Then according to (V.28):

$$
\|\psi\| \leqq \frac{1}{2 \pi \delta} \int_{\Gamma}\left\|C_{i} \hat{R}(z)\right\|\left\|C_{i} P_{i}\right\|\left\|R_{i}(z) \varphi_{i}\right\||d z|,
$$

where $C_{i}$ is defined by (II.7) with $E:=I^{+}$and

$$
\Omega_{i}:=\left\{x, d_{i}-2 \delta<d\left(x, U_{i}\right)<d_{i}-\delta\right\} .
$$

Applying Lemma II.1 to $\hat{R}$ (see Remark II.5, 3)) one has:

$$
\begin{aligned}
\left\|C_{i} \hat{R}(z)\right\|^{2} & \leqq\|\hat{R}(z)\|\left(1+e^{-\delta} b(\delta) \omega_{\delta}^{2}\|\hat{R}(z)\|\right) \\
& \leqq\|\hat{R}(z)\|\left(1+e^{-\delta / 2} b(\delta)^{1 / 2} \omega_{\delta}\|\hat{R}(z)\|^{1 / 2}\right)^{2}
\end{aligned}
$$

and since the integral over the horizontal line can be made arbitrarily small one obtains:

$$
\begin{aligned}
\|\psi\| & \leqq \frac{1}{\pi \delta}\left\|C_{i} P_{i}\right\|\left(\int_{-\infty}^{+\infty}\left(\frac{\Delta^{2}}{4}+\eta^{2}\right)^{-3 / 4} d \eta+e^{-\delta / 2} b(\delta)^{1 / 2} \omega_{\delta} \int_{-\infty}^{+\infty}\left(\frac{\Delta^{2}}{4}+\eta^{2}\right)^{-1} d \eta\right) \\
& \leqq \frac{1}{\delta}\left\|C_{i} P_{i}\right\|\left(\frac{\sqrt{2}}{\Delta^{1 / 2}}+\frac{2}{\Delta} \omega_{d} e^{-\delta / 2} b(\delta)^{1 / 2}\right),
\end{aligned}
$$

where we used the estimates $\left\|\hat{R}\left(I^{ \pm} \mp i \eta\right)\right\| \leqq\left(\frac{\Delta^{2}}{4}+\eta\right)^{-1 / 2}$ and the analogous one
for $R$. By Theorem III.1 one has:

$$
\forall \beta>0, \quad\left\|C_{i} P_{i}\right\| \leqq e^{-d\left(\Omega_{i}, U_{i}\right)} \frac{2}{\pi} b\left(\frac{\delta}{2}\right)^{1 / 2} e^{\delta / 2} \omega_{\delta}\left[|I| \beta^{-1}+2 s h^{-1}\left(\frac{2 \beta}{\Delta}\right)\right] ;
$$

then with the help of (V.25):

$$
\left\|C_{i} P_{i}\right\| \leqq \frac{4}{\pi} \frac{\sqrt{1+\delta}}{\delta} e^{3 \delta} \omega_{\delta} e^{-d_{i}}\left[4 \tilde{d}+4 \sqrt{2|I|} \frac{e^{-\tilde{d}}}{\Delta^{1 / 2}}\right]
$$

and finally using condition I.4 of Theorem I.1 one obtains:

$$
\left\|C_{i} P_{i}\right\| \leqq 5 e^{3 \delta} \omega_{\delta} \widetilde{d}^{-d_{i}}
$$


Inserting this estimate in the one for $\|\psi\|$ gives:

$$
\|\psi\| \leqq \frac{5 \sqrt{5}}{2} e^{7 \delta / 2}\left(\frac{\omega_{\delta}}{\Delta^{1 / 2}}+\frac{\omega_{\delta}^{2}}{\Delta}\right) \tilde{d} e^{-d_{i}}
$$

On the other hand according to (III.6) one has

$$
\forall \beta>0, \quad\left\|\left(1-J_{i}\right) \varphi_{i}\right\| \leqq \frac{2 \sqrt{2}}{\pi} b\left(\frac{\delta}{2}\right)^{1 / 2} e^{\delta / 2} \frac{\omega_{\delta}}{\Delta^{1 / 2}} e^{-d_{i}+2 \delta}\left[|I| \beta^{-1}+2 s h^{-1}\left(\frac{4 \beta}{\Delta}\right)\right]
$$

which leads to (following the same strategy as for the estimate of $\left\|C_{i} P_{i}\right\|$ above):

$$
\left\|\left(1-J_{i}\right) \varphi_{i}\right\| \leqq 10 e^{3 \delta} \frac{\omega_{\delta}}{\Delta^{1 / 2}} \mathfrak{d}^{-d_{\imath}}
$$

Hence (V.29, 30, 31) gives

$$
\left\|\varphi-\varphi_{i}\right\| \leqq 16 \tilde{d} e^{4 \delta} e^{-d_{i}}\left(\frac{\omega_{\delta}}{\Delta^{1 / 2}}+\frac{\omega_{\delta}^{2}}{\Delta}\right)
$$

\section{Examples and Concluding Remarks}

The simplest and most familiar example is provided by a finite number $N$ of well such that all single well potentials $V_{i}$ are identical. This holds in particular if there is a group of Euclidean transformations leaving the full potential $V$ invariant (but this is not really necessary). Then the spectrum of $H_{d}$ is identical to the spectrum of each $H_{i}$, with total multiplicity multiplied by the number of wells. Under tunneling, a given component of $\sigma\left(H_{i}\right)$ satisfying the separation requirement (I.4) gives rise to a component of $\sigma(H)$ exponentially close to it. In particular isolated eigenvalues split into groups of $N$ eigenvalues. In the semi-classical regime refined estimates on energy splittings are provided by the interaction matrix formalism of Helffer and Sjöstrand. At this point let us mention that this formalism can be recovered by our method only in situations where the isolation distance $\Delta$ in Theorem I.1 is large enough (say $O\left(\tilde{d}^{-m}\right)$ for some $m>0$ instead of $O\left(e^{-\tilde{d}}\right)$ as $\tilde{d}$ becomes large); the same remark applies in case $V$ has non-identical wells.

In fact in the semi-classical regimes, e.g. $g$ tends to zero, one has generically $\Delta=O\left(g^{p}\right)$ for some $p>0$ whereas $\widetilde{d}$ is $O\left(g^{-1}\right)$ so that conditions (I.3), (I.4) always hold for $g$ small enough. As an example of non-identical wells let us just mention the "flea on the elephant" phenomenon [JMSc1, HSj2, GGJ, Sim3], namely the fact that small perturbations of a family of identical wells, thus producing energy shifts in some wells and isolation distances as small as described by (I.3) and (I.4), are nevertheless able to produce localisation of wave-functions into some wells as a consequence of Theorem V.7.

There are many physical examples of interest with an infinity of wells. The simplest one is provided by periodic potentials. In this case our results complement those of Outassourt [O] and Simon [Sim2] (see also Harrell [Ha2]) about the size of bands in the semi-classical limit. For an arbitrary isolated eigenvalue $E_{0}$ of a given cell, satisfying again the isolation condition (I.4) for some interval $I$ containing $E_{0}, H$ has in its spectrum a band around $E_{0}$ whose size is exponentially 
small in the sense of (V.8). Actually this band spectrum appears in more general situations with an infinite number of identical wells since periodicity plays no rôle in the application of our stability theorems. An example of this is provided by the hierarchical models of Jona-Lasinio et al. [JMSc2, JMSc3]. Notice that in these symmetric situations states in $P \mathscr{H}$ are delocalized since those in $P_{d} \mathscr{H}_{d}$ are. Many interesting examples with an infinity of non-identical well are provided e.g. by quasi-periodic potentials or random potentials. There one expects that the stability properties as described e.g. by Theorem V.7 should give a key for the analysis of the spectral properties of $H$ and in particular of the localisation properties of wave-functions in the generic situation where, for a given energy range, there is only a finite number of resonant wells. An illustration of what can happen is provided again by the "flea on the elephant" phenomenon which occurs e.g. when a periodic potential is perturbed by impurities. Under small perturbations of some of the wells, just big enough to shift the single well energies by an amount larger than $2 \Delta$ as described in (I.4), the eigenvalues of the perturbed wells don't contribute any longer to the original energy band. They can either give rise to a new band, in case infinitely many wells are perturbed by the same impurities, or give isolated eigenvalues of $H$. As to the band wave-functions, they now avoid the wells having impurities whereas some new states appear associated to the impurity components of the spectrum.

As a last example involving continuous spectrum for "single-well" Hamiltonians $H_{i}$ let us just mention the situation where $\mathbb{R}^{n}$ is a union of infinite pieces of periodic media with different periodic potentials in each piece. To each homogeneous component corresponds some $H_{i}$ which has a band spectrum as we remarked before. If the separation assumption (I.4) holds for one of these bands, then it will give rise to a band in the spectrum of $H$, exponentially close to the original one with band wave-functions localized in the corresponding periodic piece. There remains a weak point in our statements about localisation, namely the fact that Theorem V.7 only says that wave-functions are uniformly small outside resonant wells. On the other hand it appears (see e.g. [JMSc4] and [HSj3]) that from the point of view of tunneling, non-resonant wells lie on the same footing as the classically forbidden regions. This suggests that strong improvement of the decay properties of Sects. II and III, hence of stability properties of Sect. V, are possible. This problem is under current investigation.

We have not discussed in this paper the shape resonance problem which is a particular example of a multiple well situation with at least one non-compact well. Since in this case the spectrum of $H_{d}$ has generically non-isolated eigenvalues in a continuum with no gaps our results do not apply directly. It is well-known that this difficulty can be dealt with using complex distortions to move away the continuous spectrum of infinite exterior wells which thus become non-resonant if some nontrapping condition is satisfied. There is now a large amount of literature on this problem [AsHa, CDS2, CDKS, HSj4, HiSig, Sig]; the suitable adaptation of the method presented here will appear in [BCD2]. 


\section{References}

[Ad] Adams, R.A.: Sobolev spaces. New York: Academic Press 1975

[Ag] Agmon, S.: Lectures on exponential decay of solutions of second order elliptic operators. Princeton, NJ: Princeton University Press 1982

[AsHa] Asbaugh, M., Harrell, E.: Perturbation theory for shape resonances and large barrier potentials. Commun. Math. Phys. 83, 151 (1982)

[BCD1] Briet, Ph., Combes, J.M., Duclos, P.: Spectral properties of Schrödinger operators in the semi-classical limit. Proc. Int. Conf. on Diff. Eqn. Math. Phys., Knowles, I., Saito, Y. (eds.). Lecture Notes in Math. Berlin, Heidelberg, New York: Springer 1987

[BCD2] Briet, Ph., Combes, J.M., Duclos, P.: On the location of resonances in the semi-classical limit III: Shape resonances (to appear)

[BCD3] Briet, Ph., Combes, J.M., Duclos, P.: Spectral stability under tunneling for Schrödinger operators, to appear in the Proc. of the Conf. on Partial Differential Equations, Holzhau (DDR) April 1988. Teubner-Text zur Mathematik

[CDS1] Combes, J.M., Duclos, P., Seiler, R.: Convergent expansions for tunneling. Commun. Math. Phys. 92, 229 (1983)

[CDS2] Combes, J.M., Duclos, P., Seiler, R.: On the shape resonances. Lecture Notes in Physics, vol. 211, p. 64. Berlin, Heidelberg, New York: Springer 1984

[CDKS] Combes, J.M., Duclos, P., Klein, M., Seiler, R.: The shape resonance. Commun. Math. Phys. 110, 215 (1987)

[GGJ] Graffi, S., Grecchi, V., Jona-Lasinio, G.: Tunneling instability via perturbation theory. J. Phys. A 17, 2935 (1984)

[Ha1] Harrell, E.: Double Wells. Commun. Math. Phys. 119, 351 (1979)

[Ha2] Harrell, E.: The band structure of a one-dimensional periodic system in a scaling limit. Ann. Phys. 75, 239 (1980)

[HSj1] Helffer, B., Sjöstrand, J.: Multiple Wells in the semi-classical limit. Commun. Part. Diff. Eq. 9, 337 (1984)

[HSj2] Helffer, B., Sjöstrand, J.: Puits multiples. II. interaction moléculaire, symetries, perturbation. Ann. Inst. H. Poincaré 2, 127 (1985)

[HSj3] Helffer, B., Sjöstrand, J.: Interaction through non-resonant Wells. Mathematishe Nachriten (1985)

[HSj4] Helffer, B., Sjöstrand, J.: Resonances en limite semi-classique, [Suppl.] Bulletin S.M.F. 114 (1986)

[HiSig] Hislop, P., Sigal, I.M.: Semi-classical theory of shape resonances in quantum mechanics. Mem. Am. Math. Soc. 78 (399), (1989)

[JMSc1] Jona-Lasinio, G., Martinelli, F., Scoppola, E.: New approach in the semi-classical limit of quantum mechanics. I. Multiple tunnelings in one dimension. Commun. Math. Phys. 80, 223 (1981)

[JMSc2] Jona-Lasinio, G., Martinelli, F., Scoppola, E.: Multiple tunneling in $d$-dimensions: a quantum particle in a hierarchical potential. Ann. Inst. H. Poincaré 43, 2 (1985)

[JMSc3] Jona-Lasinio, G., Martinelli, F., Scoppola, E.: A quantum particle in a hierarchical potential with tunneling over arbitrary large scales. J. Phys. A 17 (1984)

[JMSc4] Jona-Lasinio, G., Martinelli, F., Scoppola, E.: Tunneling in one dimension: general theory, instabilities, rules of calculation, applications. Mathematics and physics, lecture on recent results, Vol. 11, Streit, L. (ed.). World Scientific: Singapore 1986

[K] Kato, T.: Perturbation theory for linear operators. Berlin, Heidelberg, New York: Springer 1966

[MaR] Martinez, A., Rouleux, M.: Effet tunnel entre puits dégénérés. Comm. P.D.E. (1988) 13, 1157

[O] Outassourt, A.: Analyse semi-classique pour l'opérateur de Schrödinger à Potentiel Périodique. J. Funct. Anal. 72, 1 (1987)

[RS] Reed, M., Simon, B.: Methods of modern mathematical physics. II. Fourier analysis, self-adjointness. New York, San Francisco, London: Academic Press 1975 
[Sig1] Sigal, I.M.: Geometric Parametrices and the Many-Body Birman-Schwinger Principle. Duke Math. J. 50, 517 (1983)

[Sig2] Sigal, I.M.: Sharp Exponential bounds on resonance states and width of resonances. Adv. Appl. Math. 9, 127 (1988)

[Sim1] Simon, B.: Semi-classical analysis of low-lying eigenvalues. II. Tunneling. Ann. Math. 120, 89 (1984)

[Sim2] Simon, B.: Semi-classical analysis of low-lying eigenvalues. III. Width of the groundstate band in strongly coupled solids. Ann. Phys. 158, 415 (1984)

[Sim3] Simon, B.: Semi-classical analysis of low-lying eigenvalues. IV. The flea on the elephant. J. Funct. Anal. 63, 123 (1985)

Communicated by B. Simon

Received May 3, 1988; in revised form May 31, 1989 